\title{
The Effect of Balanced Analgesia with Sufentanil and Flurbiprofen Axetil in Patients with Cervical Cancer after Open Surgery
}

\author{
Tian-Xin Dong* \\ Affiliated Hospital of Chengde Medical University, Chengde 067000, Hebei Province, China \\ *Corresponding author: Tian-Xin Dong, Email: kj17539@21cn.com
}

DOI: $10.37155 / 2717-5278-2021-03-02-4$

\begin{abstract}
Objective: To study the effect of balanced analgesia with sufentanil and flurbiprefen axetil in patients with cervical cancer after open surgery. Methods: 92 patients with cervical cancer treated in our hospital from October 2018 to December 2020 were selected. All patients were treated with open surgery for cervical cancer. According to the odd even grouping method, the patients were divided into observation group and control group, with 46 cases in each group. Sufentanil was used for postoperative analgesia in the control group, and sufentanil combined with flurbiprofen axetil was used for postoperative analgesia in the observation group. Serum prostaglandin E2 (PGE2), $\beta$ Endorphins( $\beta$ - EP), cortisol (COR), 5-hydroxytryptamine (5-HT), VAS score at different time points after operation and the number of presses of patient-controlled intravenous analgesia pump (PCIA) were compared. Results: Before operation, serum PGE2, $\beta$ - Comparison of EP, Cor and 5-HT levels were compareed, $\mathrm{P}>0.05 ; 24$ hours after operation, the above indexes in the observation group were significantly lower than those in the control group $(P<0.05)$; The VAS scores of the observation group at different time points were lower than those of the control group $(P<0.05)$; The incidence of adverse reactions in the observation group was $6.52 \%$, lower than $23.91 \%$ in the control group $(P<0.05)$. Conclusion: In the postoperative analgesia of patients with cervical cancer after open surgery, the application of sufentanil combined with flurbiprofen axetil balanced analgesia can not only reduce the secretion of related factors, but also improve the analgesic effect with less adverse reactions, which is worthy of clinical reference.

Keywords: Cervical cancer; Sufentanil; Flurbiprofen axetil; Anaesthesia; Postoperative analgesia
\end{abstract}

\section{Introduction}

Open surgery for cervical cancer is traumatic, accompanied by severe pain, which brings great trauma to the body and mind of patients. Postoperative continuous intravenous analgesia can not only relieve pain, but also stabilize the respiratory and circulatory system and reduce the postoperative mortality. ${ }^{[1]}$ In clinical, opioids are often used for analgesia in clinic. Sufentanil is a commonly used as analgesic. Its analgesic effect is the strongest among opioids and has little impact on hemodynamics. If sufentanil is used alone, although the analgesic effect is good, it will affect the recovery of postoperative gastrointestinal function. ${ }^{[2]}$ Patients with cervical 
cancer undergoing laparotomy have great trauma, and the body releases more inflammation and pain related factors. Inflammatory cytokines are hyperalgesia mediators, which can activate phospholipase A2, release arachidonic acid, increase the synthesis of prostaglandin E2, cause inflammatory reaction and increase pain. The application of anti-inflammatory drugs while analgesia can inhibit the release of related factors. If the application of non steroidal anti-inflammatory drugs while opioid analgesia can reduce the synthesis of prostaglandins and promote the reduction of postoperative inflammatory response. Flurbiprofen axetil is a non steroidal anti-inflammatory drug. Taking lipid microspheres as the carrier, it can be targeted for treatment without affecting the recovery of anesthesia. The drugs gather in the incision and inflammatory sites and are hydrolyzed to flurbiprofen. The blood drug concentration is high, which can inhibit prostaglandin biosynthesis, and also inhibit the allergy of central and peripheral nervous system. It has a good analgesic effect on acute pain. ${ }^{[3]}$ The paper studies the effect of flurbiprofen axetil and sufentanil on postoperative analgesia in patients after open surgery for cervical cancer, which is reported as follows.

\section{Materials and Methods}

\subsection{General Materials}

92 patients with cervical cancer treated in our hospital were treated by open surgery. They were treated from October 2018 to December 2020. These patients were divided into two groups by odd even grouping method. The observation group $(n=46)$ and the control group $(\mathrm{n}=46)$. Observation group: age: $60-74$ years, average age $(65.43 \pm 3.81)$ years; ASA grade: Grade I 18 cases, grade II 28 cases; FIGO stage: 14 cases in stage I, 20 cases in stage II and 12 cases in stage III. Control group: age: $61-75$ years, average age $(65.36 \pm 3.79)$ years; ASA grade: Grade I 17 cases, grade II 29 cases; FIGO stage: 13 cases in stage I, 19 cases in stage II and 14 cases in stage III. The difference of general materials between the two groups met the comparability difference $(P>0.05)$.

Inclusion criteria: (1) informed research, voluntary open surgery for cervical cancer; (2) ASA grade 1-2; (3) approved by the ethics committee of the hospital.
Exclusion criteria: (1) history of allergy to the drug used in this study; (2) patients with immune system diseases; (3) combined with tumors in other parts.

\subsection{Methods}

All patients entered the operating room and their vital signs need to be monitored and open the venous access of upper limbs. General anesthesia and epidural block anesthesia was used in both groups. Before general anesthesia, epidural puncture was performed from waist 1-2 or chest 12 to waist 1 , and catheter was indwelling. Anesthesia induction: propofol $1.5 \mathrm{mg} / \mathrm{kg}$, midazolam $2 \mathrm{mg}$, cisatracurium $0.2 \mathrm{mg} / \mathrm{kg}$, epidural drug $0.5 \%$ ropivacaine. Remifentanil $(0.2 \mu \mathrm{g} / \mathrm{kg} / \mathrm{min})$ and propofol (4-7mg / kg / h) were given intraoperatively during the operation, dexmedetomidine was used to maintain anesthesia and cisatracurium was given intermittently. After operation, the epidural catheter was removed, the analgesic pump (Manufacturer: Beijing Smith Medical Instrument Co., Ltd.; Model: model 6300 CADD legacy PCA) was connected intravenously, and anesthetics were inserted into the

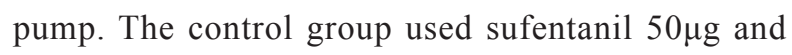
tropisetron injection $10 \mathrm{mg} / 100 \mathrm{ml}$, analgesic pump single dose $3 \mathrm{ml}$, maintenance dose $3 \mathrm{ml} / \mathrm{h}$, interval 30 minutes, when in pain, pressed the button to start the patient-controlled analgesic pump to inject drugs. The stay of PCA depended on the degree of pain of the patient. In the observation group, flurbiprofen axetil was added on the basis of sufentanil 50ug, and 200 $\mathrm{mg}$ flurbiprofen axetil injection $+10 \mathrm{ml}$ tropisetron injection were prepared into $100 \mathrm{ml}$. The setting of analgesic pump in the observation group was the same as that in the control group.

\subsection{Observation Index}

(1) levels of pain related factors and serum prostaglandin E2 (PGE2), $\beta$ Endorphins ( $\beta$ - EP), cortisol (COR), serotonin (5-HT) in 24 hours before and after operation; (2) VAS scores at $6 \mathrm{~h}, 12 \mathrm{~h}, 24 \mathrm{~h}$ and $48 \mathrm{~h}$ after operation; (3) the incidence of adverse reactions two days after operation.

\subsection{Evaluation Standards}

The pain degree of the two groups at different time after operation was scored by visual analog scoring method. The score range was $0-10$ points, and the pain 
was directly proportional to the score.

\subsection{Statistic Analysis}

Using statistical software spss24.0, "'", () denoted measurement and counting, and implementation $t$ and $\chi^{2}$ tests were performed, $P<0.05$ : there was significant difference.

\section{Results}

\subsection{The Level of Pain related Factors}

The B-EP、Cor、 $\mathrm{PGE}_{2} 、 5-\mathrm{HT}$ of two groups were compared before operation. $(P>0.05)$. The above indexes in the observation group were significantly lower than those in the control group 24 hours after operation. $(P>0.05)$. (see Table 1)

Table 1 Comparison of pain related factors between the two groups $(\bar{x} \pm s, n=46)$

\begin{tabular}{ccccccccc}
\hline \multirow{2}{*}{ Groups } & \multicolumn{2}{c}{ B-EP $(\mu \mathrm{g} / \mathrm{L})$} & \multicolumn{2}{c}{ Cor $(\mathrm{ng} / \mathrm{mL})$} & \multicolumn{2}{c}{$\mathrm{PGE}_{2}(\mathrm{pg} / \mathrm{mL})$} & \multicolumn{2}{c}{5 -HT $(\mu \mathrm{mol} / \mathrm{L})$} \\
\cline { 2 - 9 } & $\begin{array}{c}\text { Befor } \\
\text { Operation }\end{array}$ & $\begin{array}{c}\text { 24h after } \\
\text { Operation }\end{array}$ & $\begin{array}{c}\text { Befor } \\
\text { Operation }\end{array}$ & $\begin{array}{c}\text { 24h after } \\
\text { Operation }\end{array}$ & $\begin{array}{c}\text { Befor } \\
\text { Operation }\end{array}$ & $\begin{array}{c}\text { 24h after } \\
\text { Operation }\end{array}$ & $\begin{array}{c}\text { Befor } \\
\text { Operation }\end{array}$ & $\begin{array}{c}24 \mathrm{~h} \text { after } \\
\text { Operation }\end{array}$ \\
\hline $\begin{array}{c}\text { Observation } \\
\text { Group }\end{array}$ & $9.81 \pm 3.24$ & $17.21 \pm 4.15$ & $175.6 \pm 26.3$ & $243.9 \pm 31.5$ & $132.5 \pm 13.8$ & $168.3 \pm 23.9$ & $0.42 \pm 0.14$ & $0.72 \pm 0.16$ \\
$\begin{array}{c}\text { Control } \\
\text { Group }\end{array}$ & $9.71 \pm 3.19$ & $20.08 \pm 4.21$ & $174.7 \pm 25.8$ & $275.6 \pm 37.4$ & $131.6 \pm 12.7$ & $182.5 \pm 26.7$ & $0.41 \pm 0.17$ & $0.89 \pm 0.18$ \\
$t$ & 0.149 & 3.293 & 0.166 & 4.397 & 0.326 & 2.688 & 0.308 & 4.788 \\
$P$ & 0.441 & 0.001 & 0.434 & 0 & 0.373 & 0.004 & 0.379 & 0 \\
\hline
\end{tabular}

\subsection{Postoperative VAS Pain Score}

Compared with the control group, the VAS score of the observation group at different time points after operation was significantly lower $(P<0.05)$, as shown in Table 2.

Table 2 VAS pain scores of the two groups at different time points after operation $(\overline{\mathbf{x}} \pm \mathrm{s}$, scores $)$

\begin{tabular}{cccccc}
\hline Group & Cases & 6h after Operation & 12h after Operation & 24h after Operation & 48h after Operation \\
Observation Group & 46 & $4.24 \pm 0.97$ & $3.57 \pm 0.86$ & $3.04 \pm 0.52$ & $2.45 \pm 0.43$ \\
Control Group & 46 & $5.17 \pm 1.23$ & $4.25 \pm 0.95$ & $3.78 \pm 0.69$ & $3.02 \pm 0.54$ \\
$t$ & - & 4.027 & 3.599 & 5.809 & 5.6 \\
$P$ & - & 0 & 0 & 0 & 0 \\
\hline
\end{tabular}

\subsection{Incidence of Adverse Reactions}

Compared with the control group, the incidence of adverse reactions within 2 days after operation in the observation group was significantly lower $(P<0.05)$, as shown in Table 3.

Table 3 Comparison of the incidence of adverse reactions within 2 days after operation between the two groups [n(\%)]

\begin{tabular}{ccccccc}
\hline Group & Cases & Nausea and vomiting & Drowsiness & Respiratory depression & Retention of urine & Total incidence \\
\hline Observation Group & 46 & $1(2.17)$ & $2(4.35)$ & $0(0.00)$ & $0(0.00)$ & $3(6.52)$ \\
Control Group & 46 & $4(8.70)$ & $3(6.52)$ & $2(4.35)$ & $2(4.35)$ & $11(23.91)$ \\
$\chi^{2}$ & - & - & - & - & - & 5.392 \\
P & - & - & - & - & - & 0.02 \\
\hline
\end{tabular}

\section{Discussion}

Cervical cancer is a common gynecological malignant tumor in women. Cervical cancer surgery is an effective means to treat cervical cancer, but not all patients are suitable for surgical treatment. Surgery also has indications and contraindications. Although the medical level has improved in recent years and the application of various laparoscopic and hysteroscopic operations can not completely replace open surgery. Open surgery for cervical cancer is an effective method for the treatment of cervical cancer, but the continuous pain after surgery will affect the limb activities of patients, which is not conducive to early exercise and functional rehabilitation. Hyperalgesia is a strong physiological response to noxious stimuli caused by trauma or inflammation. It can also produce noxious physiological responses to non noxious stimuli, which can lead to local or systemic diseases. The central nervous system can receive stimulation signals transmitted by pain receptors. ${ }^{[4]}$ Noxious 
stimulation is often accompanied by inflammation and tissue damage. Inflammatory mediators such as prostaglandins, $\mathrm{P}$-substance, interleukin, kinin and 5-hydroxytryptamine will be released from the damaged tissue, which will make the pain in the damaged area more sensitive. The spinal dorsal horn is the cell body of peripheral pain sensing nerve fibers and the transmission point of pain information. The pain afferent nerve releases a variety of transmitters, including P-substance, excitatory amino acids, N-Methyl-D-aspartic acid, etc. After the spinal dorsal horn is combined with these mediators, the signals are transmitted to the cerebral cortex through the brainstem and thalamus. After integration, pain is generated. ${ }^{[5]}$

Nonsteroidal anti-inflammatory drugs are common analgesic drugs. Their principle is to inhibit cyclooxygenase in the central and peripheral nervous system and the transformation of leukotriene and arachidonic acid, so as to reduce the peripheral nerve sensitivity and inflammatory pain. Cyclooxygenase includes two isomers of cyclooxygenase- 1 and cyclooxygenase-2. The former is mostly expressed in tissues, which can maintain the synthesis of prostaglandins required by the body, protect the kidney, control peripheral vascular resistance and regulate platelet aggregation; inflammation. It can not only inhibit the inflammatory pain mediated by prostaglandins, reduce the pain, but also inhibit the sensitization of peripheral receptors, so as to inhibit the sensitization of peripheral nerves. It is of great significance in the prevention of chronic pain and the treatment of acute pain.

The concept of early multimodal analgesia was put forward in the early 1990s. Through the interaction of different types of analgesic drugs, it can achieve mutual superposition or synergistic analgesia. The contemporary concept of multimodal analgesia includes the combination of drugs with different action mechanisms and different analgesic techniques to block all links of pain and achieve the best analgesic effect. Multimodal analgesia can not only provide patients with good postoperative analgesia, but also reduce the adverse reactions of opioids and prevent the failure of analgesia due to individual differences. Multimodal analgesia has been widely used in clinic. The common anesthetic combination drugs are opioid analgesia and non autologous anti-inflammatory drugs. Common opioids include fentanyl, sufentanil, morphine, etc. Sufentanil is widely used in recent years. This drug has strong specific binding force to the $\mu$ receptor and strong analgesic effect. Its analgesic effect is 5-10 times than that of fentanyl. It has the advantages of fast onset, small distribution volume, short halflife and high clearance rate. It is not easy to produce drug accumulation. However, opioids have many side effects, such as nausea and vomiting, skin itching and respiratory depression, which bring bad analgesic experience to patients. Some studies have shown that some patients will have respiratory inhibition during sufentanil analgesia, and inhalation of narcotic drugs can also enhance respiratory inhibition. Nevertheless, the analgesic effect of sufentanil is stronger than its respiratory inhibition. Some scholars believe that sufentanil has little effect on respiratory rate, respiratory dynamics and ventilation, but it is more sensitive to elderly patients.

$\mathrm{PGE}_{2}$ is a metabolite of arachidonic acid and its biosynthesis requires cyclooxygenase-2, a rate limiting enzyme. A large number of studies have shown that cyclooxygenase- 2 is synthesized in a large amount in cervical cancer tissues. $\mathrm{PGE}_{2}$ is synthesized in cervical intima and epithelial cells, tumor cells also secrete $\mathrm{PGE}_{2}$, and surgical trauma will release $\mathrm{PGE}_{2}$ in a large amount. $\mathrm{PGE}_{2}$ is regulated by inhibiting the toxicity of natural killer cells and the activity of expressed $\mathrm{T}$ cells, so as to inhibit the immune surveillance function of cancer cells and promote tumor metastasis. NSAIDs can inhibit cyclooxygenase in peripheral nerve and spinal cord, reduce prostaglandin content, restore NK cell activity, reduce the expression of verifier cytokines and inhibit tumor metastasis.

Nonsteroidal anti-inflammatory drugs-NSAIDs can be divided into three categories according to the selection of cyclooxygenase: the first category is non selective cyclooxygenase inhibitors, such as flurbiprofen axetil and ibuprofen; the second is selective cyclooxygenase- 2 inhibitors, such as lornoxicam and meloxicam; the third category is specific cyclooxygenase-2 inhibitors, such as parecoxib sodium and celecoxib. No matter what kind of NSAIDs we choose, we need to weigh the advantages 
and disadvantages from the aspects of effectiveness and safety. Flurbiprofen axetil is an intravenous drug, which can nonselective inhibit cyclooxygenase-1 and cyclooxygenase-2. It has the advantages of fast onset, long duration, targeting and less side effects. It is widely used in the treatment of cancer pain and postoperative analgesia. ${ }^{[5]}$ Flurbiprofen axetil microsphere preparation is a new preparation with lipid microsphere as drug carrier. This drug is developed according to the concept of drug delivery system. Lipid microsphere can be targeted to pain tissue, enhance drug efficacy and reduce adverse reactions. ${ }^{[6]}$ The pharmacodynamic mechanism of flurbiprofen axetil injection is to release flurbiprofen axetil for lipid microspheres, which is rapidly hydrolyzed to flurbiprofen, a substance with active metabolites, which can inhibit the synthesis of prostaglandins and the activity of cyclooxygenase in arachidonic acid metabolism. Different from other non steroidal drugs, this drug has no central nervous effect and does not affect the recovery after anesthesia. It is safe and reliable. Flurbiprofen axetil has fast analgesic effect and good effect. It is an intravenous non steroid preparation. Compared with oral preparation, it reduces the stimulation to gastric mucosa and adverse reactions. ${ }^{[7]}$

24 hours after operation, the levels of B-EP, cor, PGE2 and 5-HT of patients in the observation group were significantly lower than those in the control group; the VAS score at different times after operation was also lower than that in the control group; and the incidence of adverse reactions at $2 \mathrm{~h}$ after operation was also lower than that in the control group. It can be indicated that sufentanil combined with flurbiprofen axetil has a better effect in postoperative analgesia in patients with cervical cancer.

In conclusion, sufentanil combined with flurbiprofen axetil can reduce the level of pain related factors in patients after open surgery for cervical cancer, with good analgesic effect and less adverse reactions.

\section{References}

[1]. Zhichuan Zhou, Lixia Wei, Effects of flurbiprofen axetil preemptive analgesia combined with sufentanil on postoperative pain of patients with cervical cancer and serum PGE2 and TNF- $\alpha$, Chinese Journal of Coal Industry Medicine, 2021,24(04):376-381.

[2]. Kai Ni, Lu Qian, Yijin Yu, Effects of flurbiprofen axetil and parecoxib sodium on postoperative pain and serum inflammatory stress indexes in patients after radical cervical cancer surgery, Maternal and Child Health Care of China, 2021,36(05):994-997.

[3]. Jihong Wang, Lijun Ye, Hua Zhang, Yaohui Wang, Effects of low dose ketamine combined with sufentanil on stress response and negative emotion in postoperative patients with cervical cancer, Journal of International Psychiatry, 2020,47(05):1005-1008.

[4]. Goswami Jyotirup,Manir Kazi S.,Mondal Monidipa, Mallik Suman, Das Sayan, Sen Arijit,Pal Bipasha,Das Suresh,Palit Soura,Sarkar Papai. Clinical audit of dose-escalated radical radiotherapy for advanced cervical carcinoma using a pragmatic protocol (3 fractions of $8 \mathrm{~Gy}$ HDR brachytherapy), Gynecologic Oncology Reports, 2021,37(prepublish).

[5]. Qiongli Liu, Ling Li, Fei Zhou, Zhipai Lv, Hongmei Lian, Effect of dexmedetomidine combined with sufentanil and dezocine on postoperative sleep quality in patients after radical cervical cancer surgery, Journal of Basic and Clinical Oncology, 2020,33(05):429-430.

[6]. Guoju Sun, Comparison of anesthetic effects of sufentanil and fentanyl in cervical cancer surgery, China Practical Medicine, 2020,15(17):144-145.

[7]. Galiardi-Campoy Ana Emília Brumatti,Machado Francielly Cristina, Carvalho Tamara, Tedesco Antonio Claudio, Rahal Paula,Calmon Marilia Freitas. Effects of photodynamic therapy mediated by emodin in cervical carcinoma cells, Photodiagnosis and Photodynamic Therapy, 2021,35. 